\title{
Terahertz Dielectric Resonator Antenna Coupled to Graphene Plasmonic Dipole
}

\author{
Seyed Ehsan Hosseininejad ${ }^{1}$, Mohammad Neshat ${ }^{1}$, Reza Faraji-Dana ${ }^{1}$, Sergi Abadal ${ }^{2}$, Max C. Lemme ${ }^{4}$, \\ Peter Haring Bolívar ${ }^{3}$, Eduard Alarcón ${ }^{2}$, and Albert Cabellos-Aparicio ${ }^{2}$ \\ ${ }^{1}$ School of Electrical and Computer Engineering University of Tehran, Tehran, Iran, sehosseininejad@ut.ac.ir \\ ${ }^{2}$ NaNoNetworking Center in Catalonia (N3Cat), Universitat Politècnica de Catalunya, 08034 Barcelona, Spain \\ ${ }^{3}$ Institute for High Frequency Electronic and Quantum Electronics, University of Siegen, Siegen, Germany \\ ${ }^{4}$ Faculty of Electrical Engineering and Information Technology, RWTH Aachen University, Aachen, Germany
}

\begin{abstract}
This paper presents an efficient approach for exciting a dielectric resonator antenna (DRA) in the terahertz frequencies by means of a graphene plasmonic dipole. Design and analysis are performed in two steps. First, the propagation properties of hybrid plasmonic onedimensional and two-dimensional structures are obtained by using transfer matrix theory and the finite-element method. The coupling amount between the plasmonic graphene mode and the dielectric wave mode is explored based on different parameters. These results, together with DRA and plasmonic antenna theory, are then used to design a DRA antenna that supports the $T E_{112}^{y}$ mode at $2.4 \mathrm{THz}$ and achieves a gain (IEEE) of up to $7 \mathrm{dBi}$ and a radiation efficiency of up $70 \%$. This gain is $6.5 \mathrm{~dB}$ higher than that of the graphene dipole alone and achieved with a moderate area overhead, demonstrating the value of the proposed structure.
\end{abstract}

Index Terms-dielectric resonator antenna, graphene, hybrid structures, plasmonics, terahertz.

\section{INTRODUCTION}

Terahertz band $(0.1-10 \mathrm{THz})$ wireless communications are expected to become a key technology to not only meet the increasing data rate requirements at the macroscale [1], but also to enable new applications at the micro/nanoscale such as the Internet of NanoThings [2] or software-defined metamaterials [3]. This new scenario will impose very stringent resource constraints and adverse channel conditions, among other challenges, which call for antennas and transceivers with unprecedented compactness and reconfigurability.

Graphene has been in the spotlight for the past decade due to its outstanding properties [4] and has shown promise for the implementation of terahertz antennas [5]. Thanks to its support for surface plasmon polaritons (SPPs) in the terahertz band, graphene antennas can be effectively miniaturized [6]. Another outstanding property of graphene-based antennas is tunability, which allows changing the frequency of resonance by simply varying a biasing voltage [7]. Both miniaturization and tunability, however, come at the cost of relatively low efficiency due to the significant losses of the SPP modes in graphene.

A way to address this issue is through the design of dielectric resonator antennas (DRAs). DRAs are a common technique in antenna design and is widely used in mmWave systems because of their small size, high radiation efficiency and gain, and low cost [8], [9]. Here, we propose to apply methods associated to DRAs to improve the performance of plasmonic graphene terahertz antennas in an area-efficient manner. One could, for instance, excite higher-order modes to increase the antenna gain as done in DRAs at mm-wave frequencies [9].

Hybrid plasmonic structures have been introduced to provide a better balance between mode confinement and propagation loss in waveguides operating in the visible regime with metals [10] or in the terahertz band with graphene [11]. The hybrid structure, at its most basic form, is composed of a layer of low index dielectric (Llayer), a layer of high index dielectric (H-layer), and the plasmonic material. This concept can be extrapolated to the design of antennas combining the miniaturization of plasmonics with the qualities of DRAs, but such possibility has been scarcely investigated in the literature. In [12], spoof plasmons excited by a meander slot are fed to a DRA at microwave frequencies. In [13], a metallic coplanar waveguide couples plasmons to a DRA at optical frequencies.

This paper presents, for the first time, a $\mathrm{THz}$ antenna design coupling a DRA structure to a graphene dipole using the hybrid concept mentioned above. The antenna, depicted in Fig. 1. consists of a dipole of total length $l$ and width $w$. The dipole arms are composed of five-layer graphene deposited on top of a thin Llayer, polymethylmethacrylate (PMMA) in our case. The substrate is high-resistivity Gallium Arsenide (GaAs). The dipole is assumed to be fed at its center gap of length $g$ with a photoconductive source [14] and placed below a $a \times b \times d_{H}$ rectangular resonator. The resonator is composed by a low-loss dielectric material with high permittivity (here GaAs) over a dielectric spacer (here PMMA).

In order to design an efficient antenna, a comprehensive analysis of one-dimensional and two-dimensional hybrid plasmonic structures is performed as a basic step. Section [II details the results of such analysis. Then, the dielectric resonator is designed to operate at the $T E_{y}^{111}$ and $T E_{y}^{112}$ modes when coupled to the graphene dipole based on the results of 1D-2D analysis. Section III details the design process and simulates the resulting 


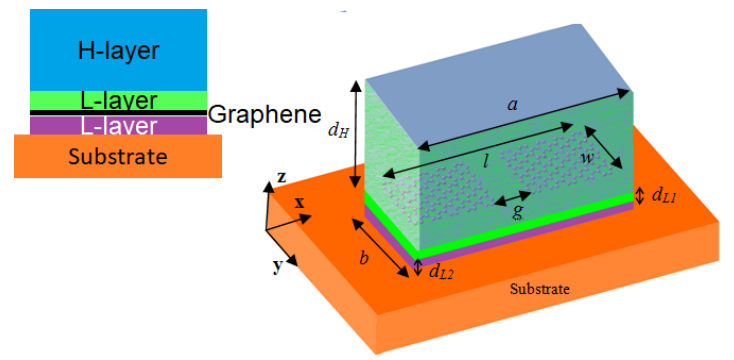

Fig. 1. Configuration of a dielectric resonator antenna coupled to a graphene plasmonic dipole.

antenna, showing that high gain radiation efficiency is achieved by exciting the DRA structure at higher-order modes. Section IV concludes the paper.

\section{ONE-DIMENSIONAL AND TWO-DIMENSIONAL HYBRID PLASMONIC STRUCTURES BASED ON GRAPHENE}

The initial step for designing a novel efficient antenna is studying the guided-wave structure related to it. For example, consider a patch antenna at microwave frequencies. In this case, knowing the propagation properties of a microstrip waveguide is very helpful to design the patch antenna appropriately. Similarly, here we first analyze one-dimensional hybrid plasmonic structures as a starting point for the design of realizable twodimensional structures.

The proposed hybrid structure, which combines the plasmonic mode and the dielectric mode, allows to exploit the benefits of both guiding mechanisms. We consider few-layer graphene with five layers as a plasmonic material, PMMA with $\epsilon_{r}=2.4$ and thickness $d_{L}$ as the L-layer and GaAs $\epsilon_{r}=12.9$ and thickness $d_{H}$ as the H-layer. Therefore, the 1D structure is PMMAGraphene-PMMA-GaAs-air. Note that the absorption losses of GaAs and PMMA are not included in the following analysis since they are small as opposed to that of graphene in the $\mathrm{THz}$ frequencies [15]. Wave is assumed to propagate in the $\mathrm{x}$-direction and to be invariant in the y-direction.

Few-layer graphene, instead of monolayer graphene, is adopted to construct the hybrid structure due to the following. Few-layer graphene has a lower propagation loss, which leads to a higher radiation efficiency. Besides, it provides a better coupling between the plasmonic mode and the dielectric mode because of the decreasing effective index of the plasmonic mode, which comes close to the index of the dielectric mode.

The approaches can be consider to perform the electromagnetic modeling of graphene. On the one hand, graphene can be modeled as a boundary condition that includes the complex surface conductivity $\sigma_{G}$ or the equivalent surface impedance $Z_{G}$, also complex [16]. On the other hand, graphene can be represented as a layer of bulk material with small thickness $d_{G}$ and equivalent complex relative permittivity $\left(\epsilon_{G}=1-j \frac{\sigma_{G}}{\omega \epsilon_{0} d_{G}}\right)$ [17]. This formalism assumes time harmonic dependence as $e^{+j \omega t}$. The results of the two modeling approaches are very similar for the conditions assumed in this work.

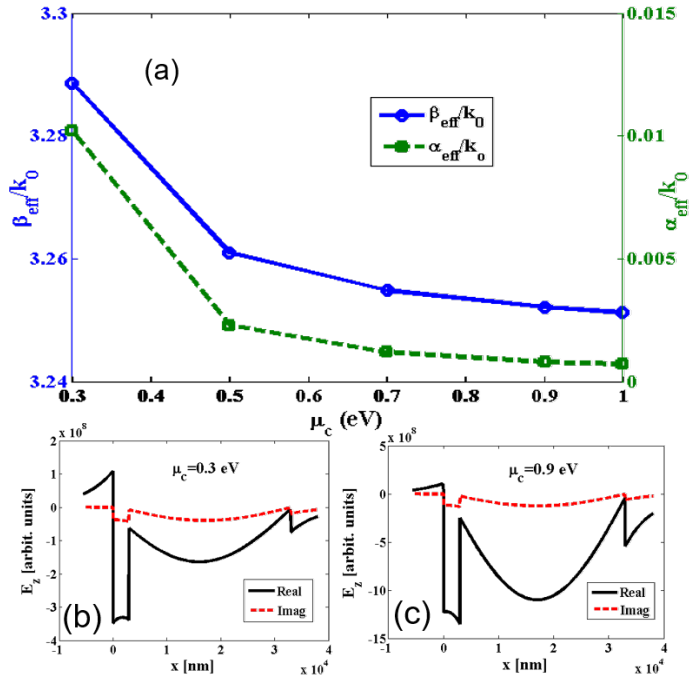

Fig. 2. Normalized propagation constant and attenuation constant hybrid 1D structure for $d_{H}=30 \mu \mathrm{m}$ and $d_{L}=3 \mu \mathrm{m}$ (top) and normal electric fields for $\mu_{c}=\{0.3,0.9\} \mathrm{eV}$ at $3 \mathrm{THz}$ (bottom left, right).

The conductivity of FLG is $N \sigma_{G}$ [15], [18], where $N$ is the number of layers $(N<6)$ and $\sigma_{G}$ is the conductivity of monolayer graphene which can be calculated by the well-known Kubo formula [19] as

$$
\begin{aligned}
\sigma_{G} & =\frac{-j}{\omega-j \tau^{-1}} \frac{e^{2} k_{B} T}{\pi \hbar^{2}}\left(\frac{\mu_{c}}{k_{B} T}+2 \ln \left(e^{-\frac{\mu_{c}}{k_{B} T}}+1\right)\right)+ \\
& +\frac{-j\left(\omega-j \tau^{-1}\right) e^{2}}{\pi \hbar^{2}} \int_{0}^{\infty} \frac{f(-\varepsilon)+f(+\varepsilon)}{\left(\omega-j \tau^{-1}\right)^{2}-4(\varepsilon / \hbar)^{2}} d \varepsilon
\end{aligned}
$$

where $\omega$ is the radian frequency, $e$ is the electron charge, $\hbar$ is the reduced Plank constant, $k_{B}$ is the Boltzmann constant, $T$ is the temperature $(T=300 \mathrm{~K}$ in this paper), $\mu_{c}$ is the chemical potential, and $\tau$ is the electron relaxation time of graphene. We consider $\tau=0.6 \mathrm{ps}$, which is reasonable considering values in related work [5]. Finally, $f(\varepsilon)=1 /\left\{1+\exp \left[\left(\varepsilon-\mu_{c}\right) /\left(k_{B} T\right)\right]\right\}$ is the Fermi-Dirac distribution function. In order to calculate the complex effective index of guided modes in the graphene-integrated structures, the formulations of transfer matrix theory provided in the previous work [20] are applied.

$1 D$ analysis: To illustrate the amount of coupling between modes, Fig. 2(a) plots the normalized propagation constant $\beta_{\text {eff }} / k_{0}$ and normalized attenuation constant $\alpha_{e f f} / k_{0}$ of the 1D structure for fixed dielectric thicknesses $\left(d_{H}=30 \mu \mathrm{m}, d_{L}=3 \mu \mathrm{m}\right)$ and variable chemical potential. Fig. 3(a) plots the same metrics for fixed chemical potential $\left(\mu_{c}=0.9 \mathrm{eV}\right)$ and variable H-layer thickness with $d_{L}=3 \mu \mathrm{m}$. The frequency is $3 \mathrm{THz}$ in both cases.

Through both plots, it is observed that the chemical potential and the H-layer thickness control the coupling strength between the plasmonic and dielectric wave modes. At small $d_{H}$ (see Fig. 2(b)) or at low $\mu_{c}$ (Fig. 3(b)), the resulting hybrid mode is more SPP-like, since most of the wave energy is concentrated at the graphene-L-layer interface, resulting in higher loss and 


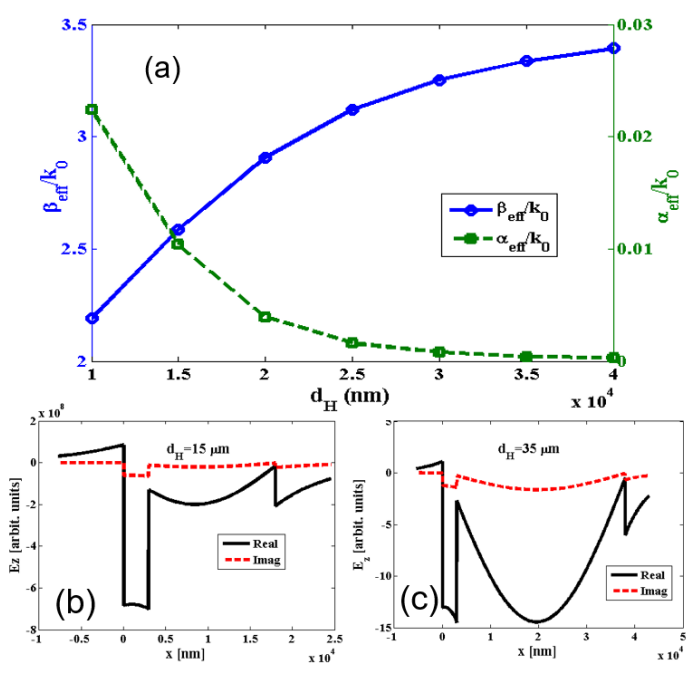

Fig. 3. Normalized propagation constant and attenuation constant of the hybrid 1D structure for $\mu_{c}=0.9 \mathrm{eV}$ and $d_{L}=3 \mu \mathrm{m}$ (top) and normal electric fields for $d_{H}=\{15,35\} \mu \mathrm{m}$ at $3 \mathrm{THz}$ (bottom left, right).

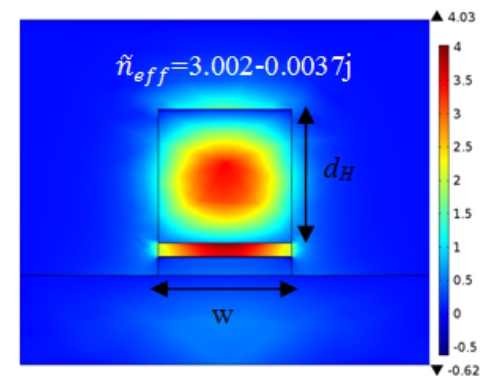

Fig. 4. Normal electric field and complex effective index of hybrid two-dimensional structure for $\mu_{c}=0.8 \mathrm{eV}, d_{H}=30 \mu \mathrm{m}, d_{L 1}=3$ $\mu \mathrm{m}$, and $d_{L 2}=4 \mu \mathrm{m}$ at $3 \mathrm{THz}$.

weaker coupling as compared to structures with large $d_{H}$ (Fig. 2, c)) or high $\mu_{c}$ (Fig. 3. (c)). Therefore, we choose sufficiently large $d_{H}$ and $\mu_{c}$ in the next design steps in order to achieve a good SPP-dielectric coupling and low loss. This design decision will lead to a more efficient antenna.

2D analysis: Let us assume a fairly high values of chemical potential $\mu_{c}=0.8 \mathrm{eV}$ and H-layer thickness $d_{H}=30 \mu \mathrm{m}$. Also, $d_{L 1}=3 \mu \mathrm{m}, d_{L 2}=4 \mu \mathrm{m}$, and $w=30 \mu \mathrm{m}$. We use the finite element method in COMSOL to obtain the normal electric field and complex effective index of the 2D structure. Fig. 4 shows the results at $3 \mathrm{THz}$. Good coupling is observed between the plasmonic mode and the dielectric mode as strong energy levels are confined in both in the L-layer region (SPP mode) and the $\mathrm{H}$-layer region (dielectric mode). It should be noted that, in this structure, we investigate the single-mode properties because the width $w$ and height $d_{H}$ of the H-layer are both smaller than half of the wavelength. However, if we increase these dimensions sufficiently, high-order modes would appear. This feature is subsequently used in the design of the antenna structure in order to excite the high-order resonating modes.

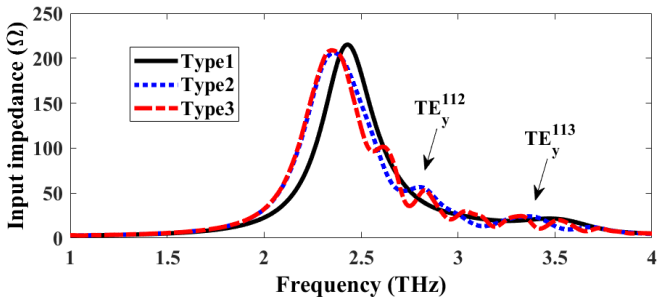

Fig. 5. Real part of input impedance for the three antenna instances (see Table I) with $\mu_{c}=0.8 \mathrm{eV}$. Position of higher-order resonances are marked for Type 2 .

\section{ANTENNA DESIGN AND EVALUATION}

As discussed in Sec. III the graphene SPP mode can be efficiently coupled to the dielectric wave mode. This idea is applied for exciting a DRA by a graphene plasmonic dipole.

Considering a rectangular DRA with relative permittivity $\varepsilon_{r}=12.9$ and with dimensions of $a \times b \times d_{H}$, the resonance frequency $f_{0}$ of the $T E_{y}^{m n p}$ mode for dielectric can be estimated from the transcendental equation as 21]

$$
k_{y} \tan \left(\frac{k_{y} b}{2}\right)=\sqrt{\left(\varepsilon_{r}-1\right) k_{0}^{2}-k_{y}^{2}},
$$

where $k_{x}^{2}+k_{y}^{2}+k_{z}^{2}=\varepsilon_{r} k_{0}^{2}, k_{x}=m \pi / a, k_{z}=p \pi / d_{H}$, and $k_{0}=2 \pi / \lambda_{0}$. It should be mentioned that the results from Eq. (2) are related to a dielectric resonator isolated in free space and it will be an approximate value for the resonance frequency in the proposed structure [21].

On the other hand, according to transmission line model for the plasmonic transverse magnetic (TM) mode on a graphene strip, the resonance frequency of a graphene dipole can be approximately predicted as

$$
l=k \frac{\lambda_{s p p}}{2}, \quad k=1,2, \ldots
$$

where $\lambda_{s p p}$ is the SPP wavelength and $k$ refers to $k$-th resonance of the dipole antenna. It is worth to note that the resonance frequency from above relation will be an approximate value because of the non-negligible capacitance of the dipole gap and also the presence of fringing fields in this geometry [22]. The first resonance $(k=1)$ of any dipole antenna has a low input impedance, and it may be seen as a short-circuit resonance (imaginary part is zero). Its second resonance $(k=2)$, where the real and imaginary parts of input impedance are large, can be interpreted as an open-circuit (OC) resonance. Contrary to in $\mathrm{RF} /$ microwave antennas, the open-circuit resonance is desired for plasmonic $\mathrm{THz}$ antennas because of the decreasing return loss obtained when connecting the antenna to photomixer $\mathrm{THz}$ sources that typically have very high impedance.

Evaluated Antennas: In order to validate the proposed design, we assume the following structural parameters: $l=20 \mu \mathrm{m}, w=5 \mu \mathrm{m}, g=2 \mu \mathrm{m}, \mu_{c}=0.8 \mathrm{eV}$, $d_{L 1}=d_{L 2}=100 \mathrm{~nm}, a=b=20 \mu \mathrm{m}$. Then, we consider three antennas with distinct DRA heights as summarized in Table I. The heights are chosen so that different representative working points of the structure 


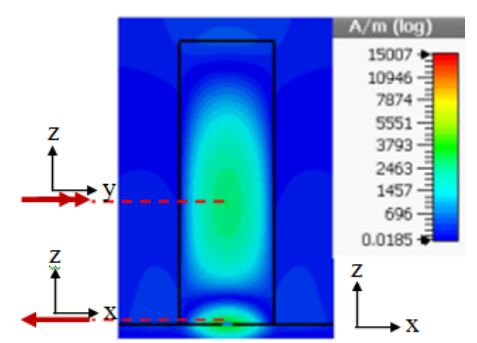
(a) $2.4 \mathrm{THz}$ (plasmonic and $T E_{y}^{111}$
modes)

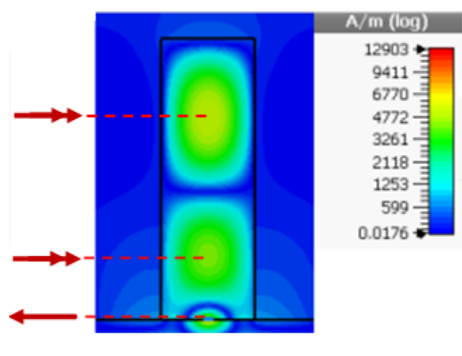

(b) $2.8 \mathrm{THz}\left(T E_{y}^{112}\right.$ mode)

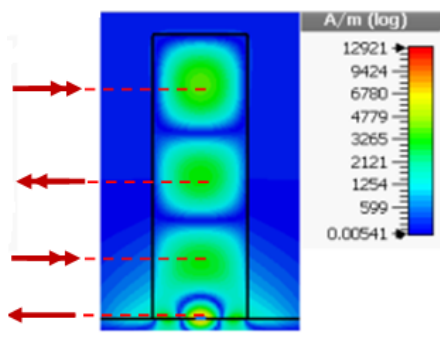

(c) $3.3 \mathrm{THz}\left(T E_{y}^{113}\right.$ mode)

Fig. 6. H-fields of the Type 2 of the designed example antenna $\left(d_{H}=60 \mu \mathrm{m}\right)$ with $\mu_{c}=0.8 \mathrm{eV}$, showing equivalent electric and magnetic dipoles at the different working points.

can be demonstrated. In the first instance (Type 1), $d_{H}$ ensures that the resonance frequency of dielectric resonator does not match with that of the graphene dipole. To this end, $d_{H}$ is small enough to consider that the radiation in the desired frequency is only caused by a pure plasmonic resonating mode (OC resonance). In the second instance (Type 2), the DRA is designed to resonate with the mode $T E_{y}^{111}$ at the $\mathrm{OC}$ resonance frequency of the graphene dipole. Consequently, the height of DRA is chosen equal to $60 \mu \mathrm{m}$. In the third instance (Type 3), we seek to make the DRA resonate at a higher-order mode $T E_{y}^{112}$ to enhance the gain in the same frequency than the OC resonance of the graphene dipole. To this end, the height of DRA is selected equal to $120 \mu \mathrm{m}$.

Methodology and results: The three antennas are modeled and simulated with CST MWS. We report results obtained with the surface impedance modeling methodology and time domain solver. Although not shown for brevity, frequency solver and permittivity modeling yielded almost identical results.

Fig. 5 illustrates the impedance matching performance. The input impedance of the antennas for the three considered case scenarios is calculated. It is observed that the OC resonance frequency of graphene dipole for Type 1 (pure plasmonic antenna) is near $2.5 \mathrm{THz}$ while it is about $2.4 \mathrm{THz}$ for Type 2 and 3 (hybrid antennas) because of the loading of the graphene dipole by the dielectric resonators. Hybrid antennas show impedance oscillations after $2.5 \mathrm{THz}$ due to the presence of higher-order modes (e.g., $2.8 \mathrm{THz}$ and 3.3 THz for Type 2).

Fig. 6 illustrates the simulated $\mathrm{H}$-field distribution of Type 2 in three frequencies. According to Fig. 5 and Fig. 6(a) $T E_{y}^{111}$ mode of DRA and OC resonance of graphene dipole occur simultaneously at $2.4 \mathrm{THz}$. $T E_{y}^{112}$ and $T E_{y}^{113}$ modes of type 2 resonate at 2.8 (Fig. 6(b) and $3.3 \mathrm{THz}$ (Fig. 6(c)), respectively. These points agree well with theoretical values from Eq. (2). It should be noticed that the plasmonic mode on the graphene dipole radiates like an electric dipole along $x$ direction and the dielectric mode on DRA acts like a $z$-oriented array of magnetic dipoles along $y$ direction, whose number depends on the mode being excited within the DRA. These equivalent electric and magnetic dipoles are

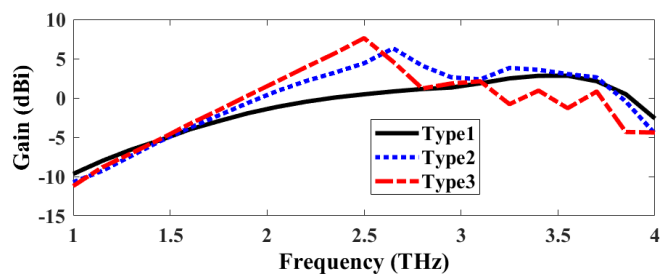

Fig. 7. Gain as a function of frequency of the designed example antenna with $\mu_{c}=0.8 \mathrm{eV}$ at $2.4 \mathrm{THz}$ for the three antenna instances.

TABLE I

SUMMARY OF ANTENNA TYPES ANALYZED IN THIS WORK.

\begin{tabular}{|c|c|l|c|c|}
\hline Structure & $d_{H}$ & Radiation mechanism & $e_{r}$ & Gain \\
\hline Type 1 & $5 \mu \mathrm{m}$ & Purely plasmonic & $65 \%$ & $0.5 \mathrm{dBi}$ \\
\hline Type 2 & $60 \mu \mathrm{m}$ & $\begin{array}{l}T E_{y}^{111} \text { DRA and } \\
\text { plasmonic }\end{array}$ & $70 \%$ & $4 \mathrm{dBi}$ \\
\hline Type 3 & $120 \mu \mathrm{m}$ & $\begin{array}{l}T E_{y}^{112} \text { DRA and } \\
\text { plasmonic }\end{array}$ & $70 \%$ & $7 \mathrm{dBi}$ \\
\hline
\end{tabular}

shown in Fig. 6

The gain (IEEE) is evaluated as a function of the frequency. Fig. 7 shows that the gain of graphene dipole loaded by DRAs (Type 2 and Type 3 ) is evidently enhanced compared with pure plasmonic antenna (Type 1). A gain of $4 \mathrm{dBi}$ and $7 \mathrm{dBi}$ is observed for the $T E_{y}^{111}$-mode DRA (Type 2) and $T E_{y}^{112}$-mode DRA (Type 3), respectively, whereas the radiation efficiency in both cases is around $70 \%$ and achieved at around 2.4 THz. This represents a clear improvement in terms of antenna performance with respect to the pure plasmonic antenna, which shows a gain of $0.5 \mathrm{dBi}$ and a radiation efficiency of $65 \%$ at around $2.5 \mathrm{THz}$. Table I summarizes the results.

Fig. 8 plots the radiation patterns of the three antennas at $2.4 \mathrm{THz}$. In Type 1, the dielectric modes do not resonate at the desired frequency and the radiation is only due to plasmonic dipole-like resonance, leading to a radiation similar to the hertzian dipole. It is also observed that the rectangular DRA operating in a higher order mode (Type 3 ) radiates a more directive pattern than the dominant mode (Type 2). The simple radiation modeling of the proposed antenna by means of an array of equivalent electric and magnetic dipoles can verify a significant increase in the directivity and gain.

In summary, several points should be generally considered to drive the design of the proposed antenna. First, design a graphene plasmonic dipole operating in 


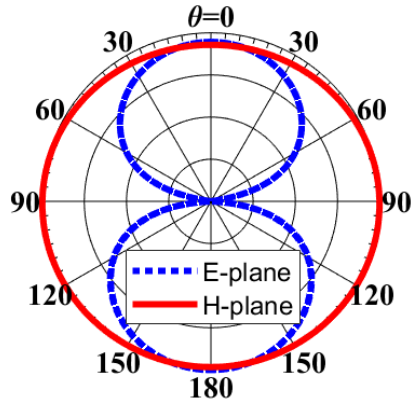

(a) Type $1\left(d_{H}=5 \mu \mathrm{m}\right)$

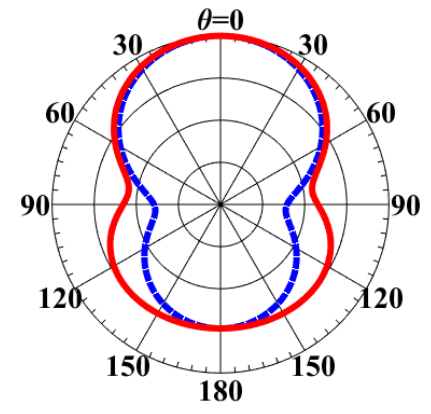

(b) Type $2\left(d_{H}=60 \mu \mathrm{m}\right)$

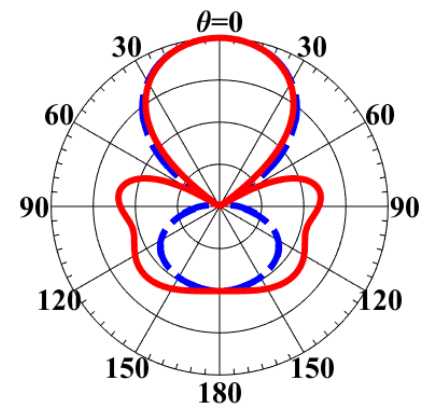

(c) Type $3\left(d_{H}=120 \mu \mathrm{m}\right)$

Fig. 8. Radiation patterns of the designed example antenna with $\mu_{c}=0.8 \mathrm{eV}$ at $2.4 \mathrm{THz}$ for the three considered case scenarios.

the OC resonance by using the approximate formula of Eq. (3) to fix the resonance frequency. Second, set the dimensions of the dielectric resonator by using the approximate formula of Eq. (2) taking into consideration the frequency and area limitations of the application. Finally, the performance of the antenna can be optimized with the help of a full-wave simulator.

\section{CONCLUSION}

In this paper, a novel $\mathrm{THz}$ antenna is proposed based on coupling of a dielectric wave mode and a plasmonic graphene mode. One-dimensional and two-dimensional analyses suggest the use of rather high chemical potential and H-layer thickness to have strong plasmonicdielectric coupling. Results of the antenna as a threedimensional structure show that an gain improvement of $6.5 \mathrm{~dB}$ is achieved by exciting the DRA at higher-order modes. The advantage of this approach for enhancing gain compared to other techniques such as the use of planar arrays is a much smaller area overhead, which is an important consideration for many applications where space limitations are of major concern.

\section{ACKNOWLEDGMENT}

This work has been partially funded by Iran's National Elites Foundation (INEF), the Spanish Ministry of Economa y Competitividad under grant PCIN-2015-012, the European Union under grant H2020-FETOPEN736876, and the German Research Foundation (DFG) under grants HA 3022/9-1 and LE 2440/3-1.

\section{REFERENCES}

[1] T. Kürner and S. Priebe, "Towards THz communications Status in research, standardization and regulation," J. Infrared, Millimeter, Terahertz Waves, vol. 35, no. 1, pp. 53-62, 2014.

[2] I. F. Akyildiz, J. M. Jornet, and C. Han, "Terahertz band: Next frontier for wireless communications," Phys. Commun., vol. 12, pp. 16-32, 2014.

[3] S. Abadal, C. Liaskos, A. Tsioliaridou, S. Ioannidis, A. Pitsillides, J. Solé-Pareta, E. Alarcón, and A. Cabellos-Aparicio, "Computing and Communications for the Software-Defined Metamaterial Paradigm: A Context Analysis," IEEE Access, vol. 5, pp. 6225-6235, 2017.

[4] A. H. Castro Neto, F. Guinea, N. M. R. Peres, K. S. Novoselov, and A. K. Geim, "The electronic properties of graphene," Rev. Mod. Phys., vol. 81, no. 1, pp. 109-162, 2009.

[5] D. Correas-Serrano and J. S. Gomez-Diaz, "Graphene-based Antennas for Terahertz Systems: A Review," FERMAT, 2017. [Online]. Available: http://arxiv.org/abs/1704.00371
[6] I. Llatser, C. Kremers, A. Cabellos-Aparicio, J. M. Jornet, E. Alarcón, and D. N. Chigrin, "Graphene-based nano-patch antenna for terahertz radiation," Photonics Nanostructures Fundam. Appl., vol. 10, no. 4, pp. 353-358, 2012.

[7] S. E. Hosseininejad and N. Komjani, "Waveguide-fed Tunable Terahertz Antenna Based on Hybrid Graphene-metal structure," IEEE Transactions on Antennas and Propagation, vol. 64, no. 9, pp. $3787-3793,2016$.

[8] A. Petosa and S. Thirakoune, "Rectangular dielectric resonator antennas with enhanced gain," IEEE Trans. Antennas Propag., vol. 59, no. 4, pp. 1385-1389, 2011.

[9] D. Hou, W. Hong, W. L. Goh, J. Chen, Y. Z. Xiong, S. Hu, and M. Madihian, "D-band on-chip higher-order-mode dielectricresonator antennas fed by half-mode cavity in CMOS technology," IEEE Antennas Propag. Mag., vol. 56, no. 3, pp. 80-89, 2014.

[10] M. Talafi Noghani and M. H. Vadjed Samiei, "Analysis and Optimum Design of Hybrid Plasmonic Slab Waveguides," Plasmonics, vol. 8, no. 2, pp. 1155-1168, 2013.

[11] S. Hosseininejad and N. Komjani, "Comparative analysis of graphene-integrated slab waveguides for terahertz plasmonics," Photonics Nanostructures - Fundam. Appl., vol. 20, pp. 1-9, 2016.

[12] A. Kianinejad, Z. N. Chen, L. Zhang, W. Liu, and C.-W. Qiu, "Spoof plasmon-based slow-wave excitation of dielectric resonator antennas," IEEE Transactions on Antennas and Propagation, vol. 64, no. 6, pp. 2094-2099, 2016.

[13] G. N. Malheiros-Silveira and H. E. Hernandez-Figueroa, "Dielectric resonator nanoantenna coupled to metallic coplanar waveguide," IEEE Photonics Journal, vol. 7, no. 1, pp. 1-7, 2015.

[14] A. Cabellos-Aparicio, I. Llatser, E. Alarcón, A. Hsu, and T. Palacios, "Use of Terahertz Photoconductive Sources to Characterize Tunable Graphene RF Plasmonic Antennas," IEEE Trans. Nanotechnol., vol. 14, no. 2, pp. 390-396, 2015.

[15] X. Zhou, T. Zhang, L. Chen, W. Hong, and X. Li, "A graphenebased hybrid plasmonic waveguide with ultra-deep subwavelength confinement," J. Light. Technol., vol. 32, no. 21, pp. 35973601, 2014.

[16] G. W. Hanson, "Dyadic greens functions and guided surface waves for a surface conductivity model of graphene," Journal of Applied Physics, vol. 103, no. 6, p. 064302, 2008.

[17] A. Vakil and N. Engheta, "Transformation optics using graphene." Science, vol. 332, no. 6035, pp. 1291-4, 2011.

[18] C. Casiraghi, A. Hartschuh, E. Lidorikis, H. Qian, H. Harutyunyan, T. Gokus, K. Novoselov, and A. Ferrari, "Rayleigh imaging of graphene and graphene layers," Nano letters, vol. 7, no. 9, pp. 2711-2717, 2007.

[19] V. P. Gusynin, S. G. Sharapov, and J. P. Carbotte, "Magnetooptical conductivity in graphene," J. Phys. Condens. Matter, vol. 19 , no. $2,2007$.

[20] S. E. Hosseininejad, N. Komjani, and M. T. Noghani, "A Comparison of Graphene and Noble Metals as Conductors for Plasmonic One-Dimensional Waveguides," IEEE Trans. Nanotechnol., vol. 14, no. 5, pp. 829-836, 2015.

[21] S. Maity and B. Gupta, "Closed form expressions to find radiation patterns of rectangular dielectric resonator antennas for various modes," IEEE Trans. Antennas Propag., vol. 62, no. 12, pp. 6524-6527, 2014.

[22] M. Tamagnone and J. Perruisseau-Carrier, "Predicting input impedance and efficiency of graphene reconfigurable dipoles 
using a simple circuit model," IEEE Antennas and Wireless

Propagation Letters, vol. 13, pp. 313-316, 2014. 